

\title{
Computation of the hindrance factor for the diffusion for nanoconfined ions: molecular dynamics simulations versus continuum-based models
}

Haochen Zhu, Aziz Ghoufi, Anthony Szymczyk, Béatrice Balannec, Denis Morineau

\section{To cite this version:}

Haochen Zhu, Aziz Ghouf, Anthony Szymczyk, Béatrice Balannec, Denis Morineau. Computation of the hindrance factor for the diffusion for nanoconfined ions: molecular dynamics simulations versus continuum-based models. Molecular Physics, 2012, 110 (11-12), pp.1107-1114. 10.1080/00268976.2011.645896 . hal-00835450

HAL Id: hal-00835450

https://hal-univ-rennes1.archives-ouvertes.fr/hal-00835450

Submitted on 26 Jun 2013

HAL is a multi-disciplinary open access archive for the deposit and dissemination of scientific research documents, whether they are published or not. The documents may come from teaching and research institutions in France or abroad, or from public or private research centers.
L'archive ouverte pluridisciplinaire HAL, est destinée au dépôt et à la diffusion de documents scientifiques de niveau recherche, publiés ou non, émanant des établissements d'enseignement et de recherche français ou étrangers, des laboratoires publics ou privés. 
Computation of Hindrance Factor of diffusion for nanoconfined ions:

Molecular Dynamic Simulations versus Continuum-based models

Haochen Zhu ${ }^{\text {a, b* }}$, Aziz Ghoufi ${ }^{\text {b, c }}$, Anthony Szymczyk ${ }^{\mathrm{a}, \mathrm{b}}$, Béatrice

Balannec $^{\mathrm{a}, \mathrm{b}}$, Denis Morineau ${ }^{\mathrm{b}, \mathrm{c}}$

${ }^{a}$ Sciences Chimiques de Rennes - UMR 6226 CNRS, Université de Rennes 1 - ENSCR, 263 Avenue du Général Leclerc, 35042 Rennes, France ; ${ }^{b}$ Université Européenne de Bretagne, France ; Institut de Physique de Rennes - UMR 6251 CNRS, Université de Rennes 1, 263 avenue du Général Leclerc, 35042 Rennes, France

*Corresponding author. Email: haochen.zhu@ecp.fr 


\title{
Computation of Hindrance Factor of diffusion for nanoconfined ions: Molecular Dynamic Simulations versus Continuum-based models
}

\begin{abstract}
We report the self-diffusion coefficients and hindrance factor of diffusion of ions into cylindrical silica nanopores. We compare with the hindered diffusion coefficients used in continuum-based models of NF. Hindrance factors for diffusion estimated from the macroscopic hydrodynamic theory were found to be in fair quantitative agreement with MD simulations for a protonated pore but they strongly overestimate diffusion inside a deprotonated pore.
\end{abstract}

Keywords: MD simulations; self-diffusion; Continuum-based models; nanofiltration

\section{Introduction}

Membrane separation processes are a class of clean processes which fit to sustainable growth and allow lowering significantly the volume of liquid waste generated in a global process compared to conventional technologies (i.e., distillation, crystallization, adsorption...). They have been becoming increasingly widespread in water treatment and wastewater reclamation and reuse applications [1-4]. Among all the membrane separation processes in liquid phase, nanofiltration (NF) is the latest one developed. Thanks to the significant improvements performed in research and development of membrane materials over the last decade, NF has today the power to solve many separation problems in an economically viable way. This promising technique has attracted increasing attention over recent years and it has already found applications in a variety of industrial sectors (e.g. dairy industry, textile industry, plating industry...).

Although NF membranes have received much attention from both academy and industry and many methods have been proposed to characterize NF membranes in order to obtain structural parameters, the transport mechanisms of solutes (especially charged solutes) through NF membranes are not fully understood [5]. The major reason is that 
the physical phenomena involved in the separation process by nanoporous membranes like those used in NF are still poorly understood. Until now, separation properties of nanofiltration membranes have been investigated only from macroscopic / mesoscopic approaches [6-8]. Although microscopic modeling is likely to give a more realistic description of transport through nanopores, few work using molecular approaches like molecular dynamic (MD) simulations to investigate the separation properties of NF membranes has been reported yet. There are basically three reasons for this: (1) NF membranes have a very complex structure and little is known about their detailed atomic structure [9] (unlike biological ion channels, the complex structure of which is now established with atomic resolution [10]); (2) The length of NF membrane pores lies most often within the range $100 \mathrm{~nm}-1 \mu \mathrm{m}$, and the current computer performances do not make possible the investigation of so large systems; (3) According to the usual volume fluxes measured experimentally with NF membranes (i.e., $50-100 \mathrm{~L} \mathrm{~h}^{-1} \mathrm{~m}^{-2}$ ), the characteristic time scale of ion passage through these materials is close to the millisecond, that is far beyond the current simulation times available from all-atom simulations. In spite of these limitations, molecular simulations are still attractive tools to get insight into nanoscale phenomena. In this work, MD simulations have been performed to investigate the dynamics properties of various aqueous electrolyte solutions (e.g. $\mathrm{NaCl}, \mathrm{NaI}, \mathrm{MgCl}_{2}, \mathrm{BaCl}_{2}$ ) and pure water confined in silica hydrophilic nanopores. Hindered diffusion inside nanopores has been also determined from the (macroscopic) hydrodynamic theory and compared to molecular simulations. 


\section{Models}

We considered a silica nanopore to get a hydrophobic cylindrical internal surface and easily functionalizable. We derived an atomic description of the silicate starting from an equilibrium structure of amorphous silica within a cubic cell of $36 \AA$ provided by Vink and Barkema [11]. Then, we applied a procedure proposed by Bródka and Zerda [12] to consider a realistic porosity within the amorphous silica. We first generated a cavity along the $z$ axis of the silica cell by removing the atoms within a cylinder of diameter $(D) 24 \AA$. From their coordination numbers, we distinguished bridging oxygens $\left(\mathrm{O}_{\mathrm{b}}\right)$ bonded to two silicon atoms from nonbridging oxygens $\left(\mathrm{O}_{\mathrm{nb}}\right)$ bonded to only one silicon and bonded to one hydrogen atom $\left(\mathrm{H}_{\mathrm{nb}}\right)$. An iterative procedure of atom $(\mathrm{O}$ and $\mathrm{Si})$ removal was applied until only tetra-coordinated silicon atoms, bonded to a maximum of two $\mathrm{O}_{\mathrm{nb}}$ 's, were present in the structure. Finally, nonbridging oxygens were saturated with hydrogen atoms to form surface hydroxyl groups. Although the silica matrix was subsequently kept rigid, rotation around the Si-O bond of the hydroxyl groups was allowed from the SHAKE constraints algorithm [13]. This procedure leads to a realistic description of the irregular inner surface of the porous silicate and of the interfacial interactions between the fluid and the matrix. The inner surface coverage of silanol groups was about $7.5 \mathrm{~nm}^{-2}$, which is correspond to highly hydrated protonated silica pore (see figure 1) [12, 14]. The high density of silanol groups was further be used to generate a deprotonated pore by removing protons of all the $\mathrm{SiOH}$ groups at the pore surface. Partial charges of the deprotoned matrix have been determined by a redistributing of the total residual charge on the framework's atoms and the partial charges are given in Table 1. This has been compared charges extracted with a first-principles calculation. For this, a combination of the Becke exchange plus Lee-Yang-Parr correlation functional and all-electron core potentials have been used. Additionally, the double- $\xi$ numerical polarization (DNP) basis set was adopted to account 
the d-type into heavier atoms and p-type polarization into hydrogen atoms. This basis is similar to the 6-31G (d,p) Gaussian-type basis set. To evaluate the charges we calculated the Mulliken population analysis. 2-4\% as difference was found between the both calculations. Although the charge density of NF membranes is expected to be much smaller, considering a strongly charged nanopore allows an easier comparison with the results obtained for a protonated nanopore (i.e., uncharged nanopore). The charges and the Lennard-Jones parameters of the different sites are summarized in Table 1.


Figure 1. Description of a protonated silica nanopore. Red indicates the oxygen atoms. The hydrogen positions are in white. Yellow are the silicon atoms. 


\begin{tabular}{|c|c|c|c|c|}
\hline & $q(e)$ & $q(e)$ & $\sigma(\AA)$ & $\varepsilon\left(\mathrm{kJ} \mathrm{mol}^{-1}\right)$ \\
\hline $\mathrm{H}_{\mathrm{nb}}$ & 0.206 & & & \\
\hline $\mathrm{O}_{\mathrm{b}}$ & -0.6361 & -0.6139 & 3.000 & 1.622 \\
\hline $\mathrm{O}_{\mathrm{nb}}$ & -0.5325 & -0.5102 & 2.700 & 1.622 \\
\hline $\mathrm{Si}$ & 1.2763 & 1.2986 & 0.000 & 0.000 \\
\hline $\mathrm{H}_{\mathrm{OSi}}$ & 0.2065 & 0.2288 & 0.000 & 0.000 \\
\hline $\mathrm{O}_{\mathrm{Si}}$ & -0.5325 & -0.5102 & 3.000 & 1.622 \\
\hline $\mathrm{H}_{\mathrm{Ob}}$ & 0.2065 & 0.2288 & 0.000 & 0.000 \\
\hline
\end{tabular}

Table 1. Charges and Lennard-Jones parameters of silica nanopore [15]. Description of labels is given in figure 1 .

As the polarizability of water molecules does not impact the ion density in the interfacial region [16] we considered here the non-polarizable TIP4P/2005 water model. This has been found to provide an impressive performance for a variety of physical properties [17]. In addition, the induced electronic polarization is known to be critical in the study of ion channels [18-20].

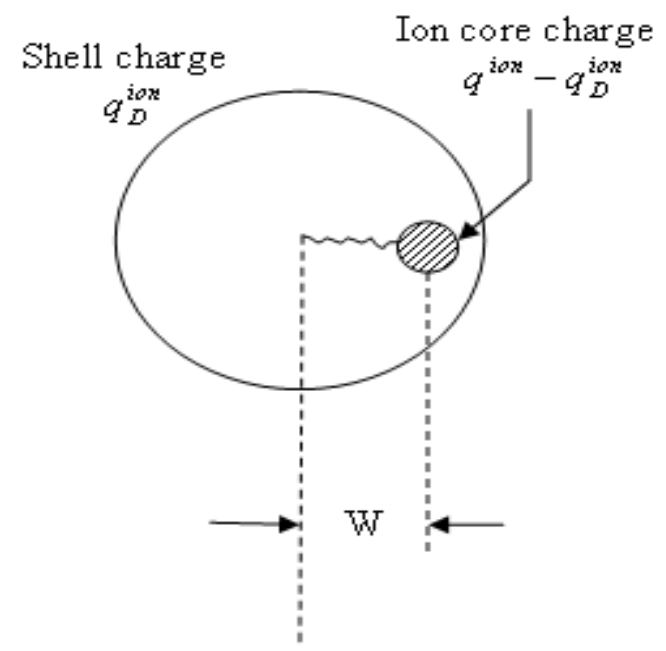

Figure 2. Scheme of core-shell model . 
In order to consider the ion polarization, we opted for the core-shell model [21] accounting for the induced polarization. This model accounts fairly well for induced polarization with limited computational cost in contrast to the induced dipole model [22] and fluctuation charge model [23] and gives the high explored length and time scales [24]. It should be stressed that the core-shell approach has never been applied to nanoconfined fluids yet. In core-shell model a polarizable atom is represented by a massive core and massless shell, connected by a harmonic spring W (see figure 2). The core and shell carry different electric charges, the sum of which equals the charge on the original atom. Thus, this ion model comprises two sites:

(1) A Drude particle attached to the ion and carrying a charge $q_{D}^{\text {ion }}$.

(2) The ion core carrying a charge of $q^{i o n}-q_{D}^{i o n}$. Both particles are coulombically screened from each other and only interact via a harmonic restoring force,

$$
U^{\text {core-shell }}=\frac{1}{2} k_{c s} r_{c s}^{2}
$$

where $r_{c s}$ is the distance between the core and shell and $k_{c s}$ is the force constant of the harmonic spring which is set to $4180 \mathrm{~kJ} \mathrm{~mol}^{-1} \AA^{-2}$ for all Drude oscillators in the system [Yu et al., 2010]. All atomic dispersion and electronic overlap effects are represented in a pairwise additive way using the Lennard-Jones potential. The core repulsion and van der Waals dispersive interactions are modeled by a Lennard-Jones interaction between the water oxygen and the ion core atom via the Lorentz-Berthelot combination rule. Lennard-Jones parameters and ions charges for both cations and anions can be found in Table 2. 


\begin{tabular}{|l|l|l|l|l|}
\hline & $q^{\text {ion }}(e)$ & $q_{D}^{\text {ion }}(e)$ & $\sigma(\AA)$ & $\varepsilon\left(\mathrm{kJ} \mathrm{mol}^{-1}\right)$ \\
\hline $\mathrm{Na}^{+}$ & +1 & -0.6876 & 2.9234 & 0.1319 \\
\hline $\mathrm{Mg}^{2+}$ & +2 & -0.4752 & 2.2528 & 0.2093 \\
\hline $\mathrm{Ba}^{2+}$ & +2 & -2.1675 & 3.1435 & 2.5121 \\
\hline $\mathrm{Cl}^{-}$ & -1 & -3.4572 & 4.9622 & 0.3013 \\
\hline $\mathrm{I}^{-}$ & -1 & -4.7331 & 5.5159 & 0.8727 \\
\hline
\end{tabular}

Table 2. Charges and Lennard-Jones parameters for ions [21]

\section{Computational procedure}

Investigating ion transport across nanopores like those of NF membranes implies developing a new modelling route to compute the correct liquid density inside nanopores. Indeed, usual methods applied in the gas phase, such as Grand Canonical Monte Carlo (GCMC) simulations, are unsuitable in the liquid phase given the high density of liquids. Indeed, in the liquid state, the chemical potential must be computed explicitly and standard techniques, like the insertion of ghost particles [25], become unsuitable because of the high probability of particle overlap (or they require excessive computational time). To overcome these difficulties, the confinement effects have been studied from Isothermal-Isosurface-Isobaric statistical ensemble which has been shown to be an efficient alternative method to Grand Canonical Monte Carlo (GCMC) simulations [26]. We considered an explicit Solid (S)/Liquid (L) interface where the volume of the porous framework is kept constant and the liquid density fluctuations are controlled by a unidirectional barostat. To build the external S/L interface we added two reservoirs of liquids surrounding the framework along the $\mathrm{z}$ axis (see figure 3 (a)). Thus, the modeled channel has two pore openings and its length is finite. 
The molecular dynamics simulations of aqueous electrolyte solutions were performed in the isothermal-isosurface-isobaric statistical ensemble using the Berendsen's barostat with periodic boundary conditions at 298 K. Dynamic of core shell particles was carried out using the Fincham's adiabatic shell dynamic [27].We set up our simulation box (see figure 2 (b)) in the $\mathrm{x}, \mathrm{y}, \mathrm{z}$ directions of $35.5,35.5,141 \AA$. All systems studied consisted of 4000 water molecules, a certain number of atoms for the pore and different types and numbers of ions. Table 3 shows the numbers of ions added in two reservoirs (see figure 3) and the corresponding salt concentrations. The LennardJones interactions were cut off at $12 \AA$ and the electrostatic interactions were computed from Ewald summation. The equations of motion were integrated using the velocity Verlet algorithm [28] with a time step of 1 fs. Data analysis was performed for the last 2 ns of simulations after 5-8 ns of equilibration. All simulations have been carried out from the modified DL-POLY package [29].



Figure 3. (a) Snapshot of the silica nanopore (b) Schematic representation of the silica pore with two reservoirs. 


\begin{tabular}{|l|ccc|}
\hline & \multicolumn{3}{|c|}{$\mathrm{NaCl}$} \\
\hline $\begin{array}{l}\text { Salt } \\
\text { concentration }\end{array}$ & $0.1 \mathrm{~mol} / \mathrm{L}$ & $0.5 \mathrm{~mol} / \mathrm{L}$ & $1 \mathrm{~mol} / \mathrm{L}$ \\
Number of cation & $5 \mathrm{Na}^{+}$ & $25 \mathrm{Na}^{+}$ & $50 \mathrm{Na}^{+}$ \\
Number of anion & $5 \mathrm{Cl}^{-}$ & $25 \mathrm{Cl}^{-}$ & $50 \mathrm{Cl}^{-}$ \\
\hline $\begin{array}{l}\text { Salt } \\
\text { concentration }\end{array}$ & $0.5 \mathrm{~mol}^{-}$ & $0.5 \mathrm{~mol}^{-} / \mathrm{L}$ & $0.5 \mathrm{~mol}^{-}$ \\
Number of cation & $25 \mathrm{Na}^{+}$ & $25 \mathrm{Mg}^{2+}$ & $25 \mathrm{Ba}^{2+}$ \\
Number of anion & $25 \mathrm{I}^{-}$ & $50 \mathrm{Cl}^{-}$ & $50 \mathrm{Cl}^{-}$ \\
\hline
\end{tabular}

Table 3. Number of ions and their corresponding concentrations in each simulation.

\section{Results and discussion}

Self-diffusion coefficient $\left(D_{s}\right)$ of water may be calculated from correlation functions, by means of Einstein relation [30]:

$$
D_{S}=\frac{1}{2 \Omega} \lim _{t \rightarrow \infty} \frac{d \mathrm{MSD}}{d t}
$$

where $\Omega$ is the dimensionality of the system (i.e., $\Omega=1$ if diffusion is considered along a single direction, $\Omega=2$ for diffusion in a plane and $\Omega=3$ for the overall diffusion through the sample volume) and MSD stands for the mean square displacement defined as,

$$
\operatorname{MSD}=\left\langle\frac{1}{N} \sum_{i=1}^{N}\left(\mathbf{r}_{i}(t)-\mathbf{r}_{i}(0)\right)^{2}\right\rangle
$$


where $\mathbf{r}_{\mathrm{i}}(t)$ denotes the position of a particle $i$ at time $t, N$ represents here the number of diffusing particles and $\langle\ldots\rangle$ the average ensemble.

Figure 4 shows the variation of the MSD of water molecules with respect to the time for water in the bulk phase and confined inside the protonated silica nanopore. Equation (2) leads to $2.30 \times 10^{-9} \mathrm{~m}^{2} \mathrm{~s}^{-1}$ for the self-diffusion coefficient of water molecules in bulk phase, which is in excellent agreement with the experimental value of $2.27 \times 10^{-9} \mathrm{~m}^{2} \mathrm{~s}^{-1}$ reported by Gillen et al. [31].



Figure 4. Means square displacement (MSD) with respect to the time for bulk and confined water molecules.

The overall self-diffusion coefficient for water inside the nanopore is much smaller than the bulk value and is found to be $0.42 \times 10^{-9} \mathrm{~m}^{2} \mathrm{~s}^{-1}$, i.e., it is reduced by more than a factor 5 with respect to the bulk self-diffusivity. This result is in qualitative agreement with recent MD simulations performed by Cazade et al. who showed that the 
self-diffusion coefficient of water is smaller inside carbon nanotubes (CNT) than in the bulk phase [32]. However, these authors obtained a diffusivity ratio (confined / unconfined) close to 0.6 with a CNT of $3 \mathrm{~nm}$ in diameter whereas we observed a much stronger effect of confinement with the silica nanopore since in our case the selfdiffusivity ratio between confined and bulk phases is less than 0.2. Although the smaller diameter of the silica nanopore under consideration in this work contributes to the stronger effect of confinement, the strong interaction between water molecules and the hydrophilic surface of the nanopore contributes significantly to the dramatic decrease in water diffusion as well. Indeed, in the case of hydrophobic materials like CNTs, two different phenomena having opposite effects are expected to affect water diffusivity. On one hand, the confinement effect related to the finite size of both the CNT and the diffusing particles hinders diffusion but, on the other hand, the weak attraction between water and CNT leads to faster diffusion of water with little resistance [33]. It can be underlined that simulations performed by Cazade et al. suggest that confinement effect in $\mathrm{CNT}$ is predominant.

The overall self-diffusion coefficients of both $\mathrm{Na}^{+}$and $\mathrm{Cl}^{-}$ions in bulk phase computed from Equation (2) for $\mathrm{NaCl}$ solutions at various concentrations are shown in Table 4

\begin{tabular}{|c|c|c|}
\cline { 2 - 3 } \multicolumn{1}{c|}{} & $D_{S, b \mathrm{Na}^{+}}\left(\mathrm{m}^{2} \mathrm{~s}^{-1}\right)$ & $D_{S, b_{C l^{-}}}\left(\mathrm{m}^{2} \mathrm{~s}^{-1}\right)$ \\
\hline $\mathrm{NaCl} 0.1 \mathrm{M}$ & $1.02 \times 10^{-9}$ & $1.47 \times 10^{-9}$ \\
\hline $\mathrm{NaCl} \mathrm{0.5} \mathrm{M}$ & $1.09 \times 10^{-9}$ & $1.56 \times 10^{-9}$ \\
\hline $\mathrm{NaCl} 1 \mathrm{M}$ & $1.05 \times 10^{-9}$ & $1.49 \times 10^{-9}$ \\
\hline
\end{tabular}

Table 4. Self-diffusion coefficients $\left(D_{S, b}\right)$ of $\mathrm{Na}^{+}$and $\mathrm{Cl}^{-}$ions in bulk $\mathrm{NaCl}$ solutions at various concentrations inferred from MD simulations and Equation (1). 
Self diffusivities of both ions are in good agreement with MD simulations published in the literature (note also the nice agreement with the experimental value of $1.66 \times 10^{-9} \mathrm{~m}^{2} \mathrm{~s}^{-1}$ for $\mathrm{Cl}^{-}$ions in a $1 \mathrm{M} \mathrm{NaCl}$ solution [34]). For example, the self diffusion coefficients of $\mathrm{Na}^{+}$and $\mathrm{Cl}^{-}$in a molar $\mathrm{NaCl}$ solution were found to be:

- $D_{S, b N a^{+}}=1.18 \times 10^{-9} \mathrm{~m}^{2} \mathrm{~s}^{-1}$ and $D_{S, b C l}=1.48 \times 10^{-9} \mathrm{~m}^{2} \mathrm{~s}^{-1}$ with the SPC water model [35]

- $D_{S, b N a^{+}}=1.1 \times 10^{-9} \mathrm{~m}^{2} \mathrm{~s}^{-1}$ and $D_{S, b C l}=1.3 \times 10^{-9} \mathrm{~m}^{2} \mathrm{~s}^{-1}$ with the SPC/E water model [36]

- $D_{S, b N a^{+}}=1.1 \times 10^{-9} \mathrm{~m}^{2} \mathrm{~s}^{-1}$ and $D_{S, b C l}=1.5 \times 10^{-9} \mathrm{~m}^{2} \mathrm{~s}^{-1}$ with the RPOL water model [36]

It should be noted, however, that these values are somehow smaller than the usual diffusion coefficients considered in NF models which correspond to transport quantities associated with an imposed concentration gradient. The reason for the discrepancy between the self-diffusivity and the "transport" diffusivity is that the former is related to the single-particle dynamics at equilibrium in the absence of an external field [32] whereas the second one describes the collective motion of particles under an external field [37]. In order to overcome (at least partially) this difficulty we shall further discuss on self diffusivity ratios instead of single value of self diffusion coefficients. For example, the mean ratio $D_{S, b N a^{+}} / D_{S, b C l}$ inferred from our simulations is equal to 0.70 (see Table 4). This result is in good agreement with the same ratio computed from usual "transport" diffusion coefficients, which is equal to 0.66 [38].

The overall self diffusion coefficient of ions inside silica nanopores (both uncharged and negatively charged) were computed for the various electrolyte solutions 
under consideration. The ratios between confined and bulk self diffusivities $\left(D_{S, p} / D_{S, b}\right)$ inferred from MD simulations are collected in Table 5 and compared with the hindrance factors for diffusion $\left(K_{i, d}=D_{i, p} / D_{i, b}\right)$. These are equivalent to the ratio between pore and bulk "transport" diffusion coefficients and correspond to the macroscopic quantities used in current NF models to account for finite size effects on solute diffusion through NF membranes [39, 40]. Several approximate analytical expressions of $K_{i, d}$ derived using the centerline approximation are available in the literature [41]. In this work, we have used the approximate equations derived by Bungay and Brenner [42] which are applicable over the entire range of the solute-to-pore size ratio [43],

$$
K_{i, d}=\frac{6 \pi}{K_{i, t}}
$$

where $K_{i, t}$ is defined as:

$$
K_{i, t}=\frac{9}{4} \pi^{2} \sqrt{2}\left(1-\lambda_{i}\right)^{-5 / 2}\left[1+\sum_{n=1}^{2} a_{n}\left(1-\lambda_{i}\right)^{n}\right]+\sum_{n=0}^{4} a_{n+3} \lambda^{n}
$$

with $\lambda_{i}=r_{i, \text { Stokes }} / r_{\mathrm{p}}\left(r_{i, \text { Stokes }}\right.$ is the Stokes radius of ion $i$ and $r_{p}$ is the pore radius $), a_{1}=-$ $73 / 60, a_{2}=77.293 / 50.400, a_{3}=-22.5083, a_{4}=-5.6117, a_{5}=-0.3363, a_{6}=-1.216, a_{7}=$ 1.647. 


\begin{tabular}{|c|c|c|c|c|}
\hline Bulk solution & $D_{S, p+} / D_{S, b+}$ & $K_{+, d}$ & $D_{S, p-} / D_{S, b-}$ & $K_{-, d}$ \\
\hline $\mathrm{NaCl} 0.1 \mathrm{M}$ & - & 0.69 & - & 0.79 \\
\hline $\mathrm{NaCl} 0.5 \mathrm{M}$ & 0.52 & 0.69 & 0.67 & 0.79 \\
\hline $\mathrm{NaCl} 1 \mathrm{M}$ & 0.52 & 0.69 & 0.62 & 0.79 \\
\hline $\mathrm{NaI} 0.5 \mathrm{M}$ & - & 0.69 & 0.50 & 0.79 \\
\hline $\mathrm{MgCl}_{2} 0.5 \mathrm{M}$ & 0.39 & 0.44 & 0.67 & 0.79 \\
\hline $\begin{array}{c}\mathrm{BaCl} \\
20.5 \mathrm{M}\end{array}$ & 0.37 & 0.52 & 0.61 & 0.79 \\
\hline $\begin{array}{c}\mathrm{NaCl} 1 \mathrm{M} \\
(\text { deprotonated }\end{array}$ & 0.15 & 0.69 & - & 0.79 \\
pore) & & & & \\
\hline
\end{tabular}

Table 5. Comparison between hindrance factors for diffusion $K_{+, d}$ and $K_{-, d}$ (computed from Equations (4) and (5)) and the ratios between confined and bulk self diffusivities for cations $\left(D_{S, p_{+}} / D_{S, b+}\right)$ and anions $\left(D_{S, p-} / D_{S, b-}\right)$ inferred from MD simulations.

MD simulations indicate a slower diffusion for all ions under confinement. The values of $D_{S, p} / D_{S, b}$ we obtained for monovalent ions confined in the protonated nanopore are close to those of Cazade et al. who reported $D_{S, p} / D_{S, b}$ ranging from $\sim 0.55$ to 0.65 for $\mathrm{Na}^{+}, \mathrm{F}^{-}, \mathrm{Cl}^{-}, \mathrm{Br}^{-}$and $\mathrm{I}^{-}$ions confined inside a CNT of $3 \AA$ in diameter [32]. This suggests that the chemical structure of the internal surface of the nanopore affects much more strongly the dynamics of water molecules than that of ions.

For the protonated pore, the magnitude of confinement effect follows the series single-charged anions < single-charged cations < double-charged cations. Interestingly, hindrance factors for diffusion $\left(K_{i, d}\right)$ estimated from the macroscopic hydrodynamic theory (Equations (3) and (4)) are in qualitative agreement with molecular simulations. From a quantitative point of view, although the macroscopic approach is found to 
underestimate the impact of confinement on ion diffusion, a rather fair agreement is obtained with diffusivity ratios inferred from MD simulations.



Figure 5. Radial distributions of $\mathrm{Na}^{+}$and $\mathrm{Cl}^{-}$ions inside the deprotonated nanopore (bulk concentration: $1 \mathrm{M}$ ).

On the other hand, the macroscopic approach strongly overestimates the diffusion of $\mathrm{Na}^{+}$ions inside the negatively charged nanopore with respect to MD simulations. Actually, Equations 3 and 4 used to compute $K_{i, d}$ were derived assuming neutral solutes diffusing along the pore axis (centerline approximation) of an uncharged pore [41]. As a result, these equations do not allow accounting for the effect of long-range electrostatic interactions on the diffusion of ions through charged nanopores (that is why they lead to the same value of $K_{i, d}$ for both uncharged and charged pores; see Table 5). Dechadilok and Deen have recently investigated the influence of electrostatic interactions on hindered diffusion of charged solutes inside charged pores by means of finite elements techniques [39]. Although their work highlights that electrostatic interactions between 
the diffusing solute and the pore walls can noticeably decrease the intrapore diffusivity, it must be stressed, however, that Dechadilok and Deen considered a centerline approximation, which is clearly inappropriate in our case. Indeed, figure 4 which shows the radial distributions of ions inside the deprotonated nanopore clearly puts in evidence that $\mathrm{Na}^{+}$ions are mainly located at the surface of the charged nanopore while they are almost absent of the central region of the nanopore. This preferential location of $\mathrm{Na}^{+}$ ions results from the strong attractive interaction between $\mathrm{Na}^{+}$ions and $\mathrm{SiO}^{-}$surface sites (the radial charge distribution of the deprotonated nanopore is shown in figure 6). This latter is also responsible for the large decrease in the intrapore diffusivity of $\mathrm{Na}^{+}$ reported in Table 5. It can be noted that no results about the diffusion of $\mathrm{Cl}^{-}$ions inside the charged nanopore are reported in Table 5 since $\mathrm{Cl}^{-}$ions were found to be totally excluded from the pore by the high negative surface charge density (see figure 5).

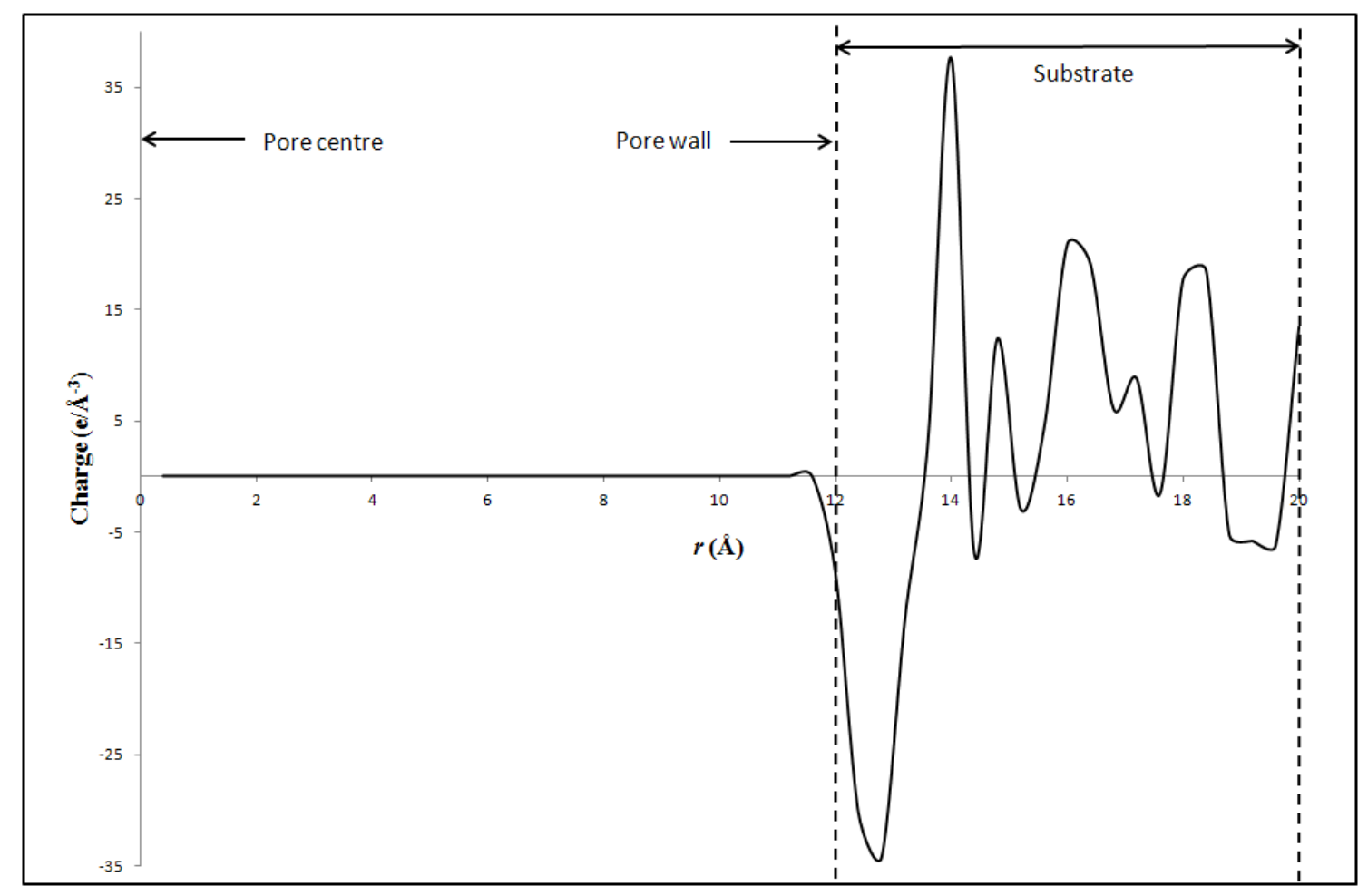

Figure 6. Radial charge distribution of deprotonated nanopore 


\section{Conclusion}

In this work, we investigated the dynamics properties of confined electrolyte solutions from the analysis of the mean square displacement versus time of both water molecules and ions. The self diffusivity of water molecules was found to be severely hindered by confinement. The same qualitative conclusion was obtained for ions. For the protonated (i.e., uncharged) pore, the magnitude of the confinement effect was found to follow the series single-charged anions < single-charged cations < double-charged cations. Interestingly, it was shown that hindrance factors for diffusion estimated from the macroscopic hydrodynamic theory give the same sequence and are even in rather fair quantitative agreement with MD simulations. On the other hand, the macroscopic approach was found to strongly overestimate the self diffusivity of ions confined inside the protonated (i.e., negatively charged) silica nanopore. This is mainly due to the fact that available analytical expressions of hindrance factors were derived by assuming neutral solutes diffusing through uncharged pores. The electrostatic interactions between the ions and the pore walls are therefore not taken into account through these approximate relations.

\section{Acknowledgements}

The authors are grateful to the EU commission (FEDER), the "Conseil Régional de Bretagne" and the "Université Européennes de Bretagne" for their financial support through the research program EPT-MOMEN. 


\section{References}

[1] M. E. Carrín, M.B. Buglione and J.E. Lozano, European Congress of Chemical Engineering, 2007.

[2] H.S. Alkhatim, M.I. Alcaina, E. Soriano, M.I. Iborra, J. Lora, and J. Arnal, Desalination 119, 177 (1998).

[3] K. Auddy, S. De and S. DasGupta, Sep. Purif. Technol. 40, 31 (2004).

[4] S.B. Sadr Ghayenia, P.J. Beatsona, R.P. Schneiderb and A.G. Fanea, Desalination 116, 65 (1998).

[5] K.Y. Wang and T. Chung, J. Membr. Sci. 247, 37 (2005).

[6] A. Szymczyk, H. Zhu and B. Balannec, J. Phys. Chem. B 114, 10143 (2010).

[7] H. Zhu, A. Szymczyk and B. Balannec, J. Membr. Sci. 379, 215 (2011).

[8] W.R. Bowen, A.W. Mohammad and N. Hilal, J. Membr. Sci. 126, 91 (1997).

[9] E. Harder, D.E. Walters, Y.D. Bodnar, R.S. Faibish and B. Roux, J. Phys. Chem. B 113, 10177 (2009).

[10] E. Tajkhorshid, P. Nollert, M. Jensen, L.J.W. Miercke, J. O'Connell, R.M. Stroud and K. Schulten, Science 296, 525 (2002).

[11] R.L.C. Vink and G.T. Barkema, Phys. Rev. B 67, 245201 (2003).

[12] A. Bródka and T.W. Zerda, J. Chem. Phys. 104, 6319 (1996).

[13] J-P. Ryckaert, G. Ciccotti and H.J.C. Berendsen, J. Comput. Phys. 23, 327 (1977).

[14] J. Puibasset and R.J.-M. Pellenq, J.Chem. Phys. 122, 094704 (2005).

[15] A. Ghoufi, D. Morineau, R. Lefort and P. Malfreyt, J. Chem. Theory Comput. 6, 3212 (2010).

[16] Y. Levin, Phys. Rev. Lett. 102, 147803 (2009).

[17] C. Vega and J.L.F. Abascal, Phys. Chem. Chem. Phys. DOI: 10.1039/c1cp22168j.

[18] B. Roux, Chem. Phys. Lett. 212, 231 (1993).

[19] T.W. Allen, O.S. Andersen and B. Roux, Biophys. J. 90, 3447 (2006).

[20] D. Bucher, L. Guidoni, P. Maurer and U. Rothlisberger, J. Chem. Theory Comput. 5, 2173 (2009).

[21] H. Yu, T.W. Whitfield, E. Harder, G. Lamoureux, I. Vorobyov, V. M. Anisimov, A. D. MacKerell, Jr. and B. Roux, J. Chem. Theory Comput. 6, 774 (2010). 
[22] D. Elking, T. Darden and R.J. Woods, J Comput Chem. 28, 1261 (2007).

[23] Y. Ma and S.H. Garofalini, J. Chem. Phys. 124, 234102 (2006).

[24] G. Lamoureux and B. Roux, J. Phys. Chem. B 110, 3308 (2006).

[25] B. Widom, J. Chem. Phys. 39, 2802 (1963).

[26] A. Ghoufi, D. Morineau, R. Lefort, I. Hureau, L. Hennous, H. Zhu, A. Szymczyk, P. Malfreyt and G. Maurin, J. Chem. Phys. 134, 074104 (2011).

[27] D. Fincham and P.J. Mitchell, J. Phys. Condens. Matter 5, 1031 (1993).

[28] M.P. Allen and D.J. Tildesley, Computer Simulation of Liquids, Oxford University Press Inc. New York, 1987.

[29] T.R. Forester and W. Smith, DLPOLY, CCP5 Program Library (Daresbury Laboratory, UK, 2001).

[30] I. C. Bourg and G. Sposito, J. Colloid Interface Sci. 360, 701 (2011).

[31] K.T. Gillen, D. C. Douglass and M.J.R. Hoch, J. Chem. Phys. 57, 12 (1972).

[32] P.A. Cazade, J. Dweik, B. Coasne, F. Henn and J. Palmeri, J. Phys. Chem. C 114, 12245 (2010).

[33] R. Srivastava, H. Docherty, J. K. Singh and T.Cummings, J. Phys. Chem. C 115, 12448 (2011).

[34] D. Laage and J. T. Hynes, Proc. Nat. Acad. Sci. USA 104, 11167 (2007).

[35] S. Koneshan and C. Rasaiah, J. Chem. Phys. 113, 18 (2000).

[36] J. Sala, E. Guardia and J. Marti, J. Chem. Phys. 132, 214505 (2010).

[37] T. Ala_Nissila, R. Ferrando and S.C. Ying, Adv. Phys. 51, 949 (2002).

[38] D.R. Lide, Handbook of Chemistry and Physics, CRC Press, 2000.

[39] P. Dechadilok and W.M. Deen, J. Membr. Sci. 336, 7 (2009).

[40] A. Szymczyk and P. Fievet, J. Membr. Sci. 252, 77 (2005).

[41] W.M. Deen, AIChE J. 33, 1409 (1987).

[42] P.M. Bungay and H. Brenner, Int. J. Multiphase Flow 1, 25 (1973).

[43] T. R. Noordman and J.A. Wesselingh, J. Membr. Sci. 210, 227 (2002). 